\title{
3D Perception of a 2D Image Using Displacement Map
}

\author{
Kaushal Patel \\ Department of Mathematics, Veer Narmad South Gujarat University, Surat, India
}

Corresponding author: kbpatel@vnsgu.ac.in

\begin{abstract}
The 3D image gives the experience of the invisible dimension of the 2D image. Many researchers work for a display that projects $3 \mathrm{D}$ images in a way that makes them visible from all angles and does not require extra accessories. In this paper, we propose an algorithm to construct a 3D image form a 2D image. In which two major techniques are utilized namely segmentation and displacement map. Segmentation technique is used to classify foreground and background objects which plays an important role in 3D imaging. Our focus is only on the foreground of an image because that relates and enhances the overall composition of an image. Displacement map technique is used for constructing the 3D perception of foreground object that will mimic the quality predictions of $3 \mathrm{D}$ perception.
\end{abstract}

Keywords: 2D image, 3D image, segmentation, displacement map

In an image, objects low or directly in front of us are perceived as closest to us and are in the foreground. They are larger, clearer, and brighter than those "behind" them. Objects at a medium distance are perceived as mid-ground; they are in the middle of the frame. Objects farthest from us, in the background, are usually higher in the picture; they seem less clear, and their colors are less intense than those in both the foreground and middle ground.

To understand an image, the first step is to identify foreground and background so that we can focus on an interested object. In computer vision application, object detection is fundamental and most important steps for video analysis ${ }^{[9]}$. Therefore, in 3D modeling, foreground and background have its own importance. 3D modeling is a technology through which three-dimensional models of any given object can be created. The product design process has become easy with $3 \mathrm{D}$ modeling as we can create three-dimensional models virtually for any object no matter how gigantic or micro it is. This very fact helps to create an exquisite design for any product (e.g. orthodontic diagnosis) ${ }^{[4]}$.

3D means three-dimensional, that is, something that has a width, height, and depth (length). Our physical environment is three-dimensional and we move around in 3D every day ${ }^{[7]}$. Humans are able to perceive 
the spatial relationship between objects just by looking at them because we have 3D perception, also known as depth perception. As we look around, the retina in each eye forms a two-dimensional image of our surroundings and our brain processes these two images into a $3 \mathrm{D}$ visual experience. There are high expectations for the further development of three-dimensional display technology ${ }^{[11]}$.

The accuracy which 3D models contain is unparalleled and no other conventional modeling or visualizing approach can be as accurate as 3D modeling.

In this work, we focus only on the foreground of an image. We use a foreground that relates and enhances the overall composition of an image. we will achieve by creating depth and a dynamic visual relationship between nearer and farther elements using displacement map. Having something of visual interest that is closer than your main subject adds depth and compositional power. The goal of 3D perception of a 2D image using Displacement map is to design models that are able to generate the 3D perception of 2D image and predict image accurately based on primary information. An ideal objective of this method should be able to mimic the quality predictions of 3D perception of an average human observer. Based on the availability of a reference image which is considered to be distortion-free and has a perfect quality.

\section{Literature Review}

In the field of robotics and face recognition, foreground and background play an important role to identify the objects. Especially AI (Artificial Intelligence) is a rapidly growing field of the current century and therefore, the $3 \mathrm{D}$ vision has become the area of interest of many researchers.

The fundamental difference between $2 \mathrm{D}$ and $3 \mathrm{D}$ vision is the inclusion of the third coordinate - depth $^{[3]}$. This information can be acquired by various means ranging from stereoscopy (use of two specifically aligned cameras) to laser scanning of the environment, while each of these techniques has their own advantages and disadvantages ${ }^{[1,8]}$.

In industry and manufacturing, 3D vision systems provide unprecedented precision and flexibility in control, measurement, and quality inspection. While these areas require different approaches to hardware construction and methods of 3D image acquisition, most of the image processing algorithms are used universally ${ }^{[3]}$.

As an important part of digital image processing technology, the 3D reconstruction technology has been widely applied in medical research, remote sensing and telemetry and virtual reality, etc. ${ }^{[10,12]}$. With the

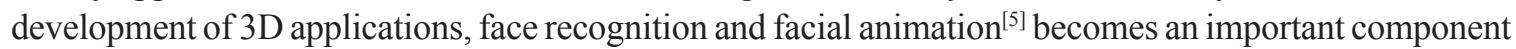
of $3 \mathrm{D}$ content production ${ }^{[2]}$. Another exploring field is 3D printing technology. 3D printing has been a great impact in many industries such as medical science, engineering, aerospace, education and many more.

The results of this work are produced in Matlab. Matlab is a great tool in the field of image processing to accomplish these tasks and it is available all over the world nowadays.

\section{Algorithm}

Algorithm involves following steps:

Image Pre-processing: Pre-processing is applied to images at the lowest level of abstraction and its aim is to reduce undesired distortions and enhance the image data which is useful and important for further processing. It is usually necessary and required for improving the performance of image processing 
methods like image transform, segmentation, feature extraction, and fault detection. This Step is focused on filtering and intensity adjustment as pre-processing methods.

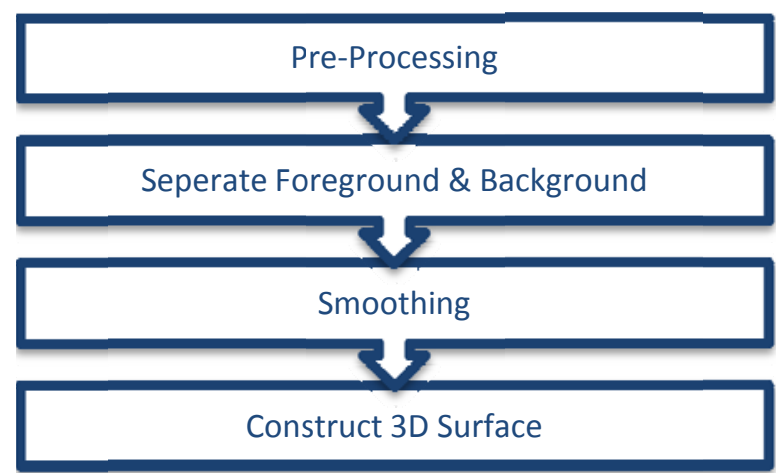

Foreground and Background: With the acquisition of an image, the first step is to distinguish objects of interest from the background. The foreground objects revealing by separating the background is, in general, a subsequent phase of image segmentation. Image segmentation is a process of identifying and then grouping region-forming pixels, satisfying single or multiple constraints ${ }^{[6]}$.

Segmentation is the process of assigning each pixel in the source image to two or more classes. If there are more than two classes, then the usual result is several binary images. Segmentation assigns pixels to foreground or background based on greyscale intensity. One way to extract the objects from the background is thresholding.

The thresholded (binary) image $g(x, y)$ is defined as,

$$
g(x, y)= \begin{cases}a & \text { if } f(x, y)>T \\ b & \text { if } f(x, y) \leq T\end{cases}
$$

Where $a$ and $b$ are distinct intensity. Then any image point $(x, y)$ at which $f(x, y)>T$ is called an object (or foreground) point which is labelled 1; otherwise, the point is called a background point which is labelled 0 .

Construct 3D surface: Displacement map or height mapcan be used to construct a polygonal "mesh" based on the intensity values of pixels in an image.

An image can be defined as $f(x, y)$. Take a small square of four pixels from this image. Each one has an $x$ and $y$ coordinate, taken straight off the image. The lower left pixel is $(0,0)$ and the upper right is $(1,1)$. Our goal is to generate two triangles in 3D space from these four pixels. In the diagram below, each of our four pixels is marked as an ' $x$ ' and we can draw two triangles. 
Each pixel has an $\mathrm{X}$ and $\mathrm{Y}$ already. So, we can already write down the vertices for two 2D triangles:

Triangle 1: $(0,0)(0,1)(1,1)$

Triangle 2: $(0,0)(1,1)(1,0)$

Now, to turn these 2D coordinates into 3D coordinates, take each of our four pixels and calculate its brightness. We use this brightness, scaled into some appropriate range, as the $\mathrm{Z}$ coordinates.

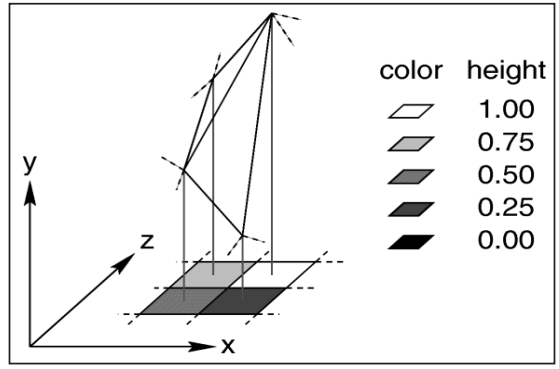

Fig. 1: Horizontal displacement of an intensity

A square of four pixels and calculate the intensity of each pixel. Then, use these four intensity values as the four $\mathrm{Z}$ coordinates. We have two triangles in 3D space generated from four pixels. We do over the whole image and finally get 3D image as shown in Fig. 1.

\section{Experimental Results}

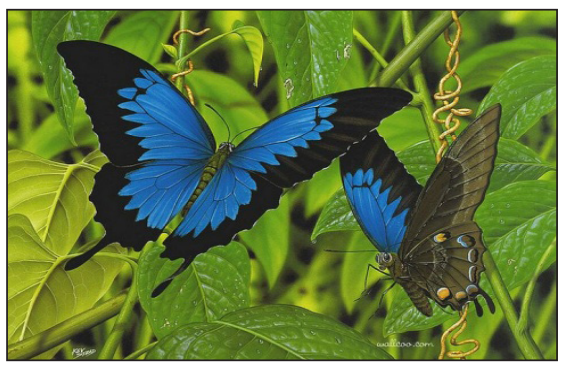

(a)

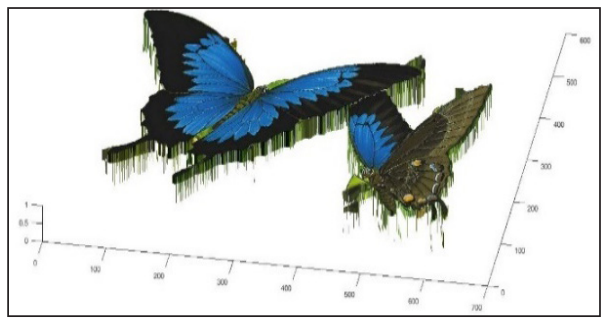

(c)

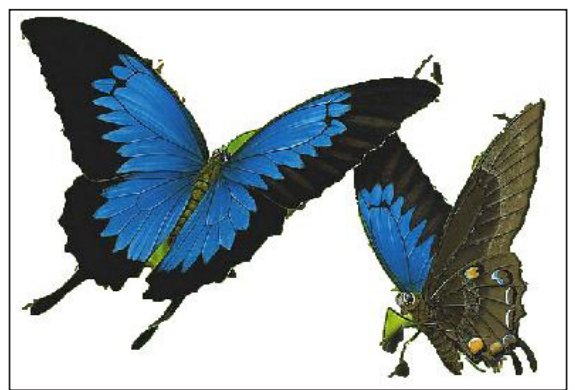

(b)

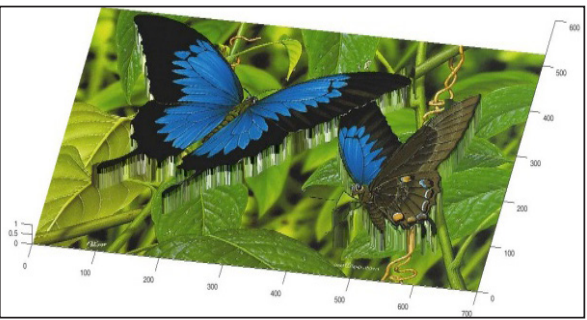

(d)

Fig. 2: (a) Original Image (b) Foreground Image (c) Construction of Foreground (d) Final 3D perception of an Image 


\section{CONCLUSION}

We are unable to include 3D perception in every photograph that we take. We find that foregrounds add considerable depth to composition image. When we use a foreground, it helps to establish a visual relationship between the bottom of the photograph and the top, which is important for leading the viewer's eye into the scene. This technique provides very detailed and valuable information about the environment or examined objects. 3D perception technique is useful to understand the characteristics of an object from a single $2 \mathrm{D}$ image. In an image, this technique produces image quality measurements which are more relevant to the volume spatial content of an integral image than conventional reliability on optically encoded spatial distribution. This work enables us to visualized and understand the three-dimensional object behind a two-dimensional image. The vast application of the algorithm can be explored further, which large potential to be used in a lot more areas than just for an aesthetic purpose.

\section{REFERENCES}

1. Pirjan A. and Petroşanu, D. "The Impact of 3D Printing Technology on The Society and Economy".

2. Bisoyi, A.A. and Das, M. 2015. "A Survey and Comparative Analysis of Various 3D Reconstruction Methods", International Journal of Advanced Research in Computer Science and Software Engineering, 5(3).

3. Varga, M. and Jadlovský, J. 2016. “3D imaging and image processing - literature Review”, Scientific Conference of Young Researchers - FEI TU of Košice, May.

4. Karatas, O.H. and Toy, E. 2014. "Three-dimensional imaging techniques: A literature review", European Journal of Dentistry, 8(1).

5. Gnanamuthu, P. and Balasubramanian, S. 2010. "Literature Review of Facial Modeling and Animation Techniques", International Journal of Graphics and Multimedia, 1(1): 1 - 14.

6. Gonzalez, R.C. and Woods R.E. 2007. Digital Image Processing. Prentice-Hall, Englewood Cliffs.

7. Vyas, S.D., Patelia, R.M. 2015. "A Review on the Role of 3D Technology in the Field of Industry and Entertainment", International Journal of Advance Research in Computer Science and Management Studies, 3(2): 109-115.

8. Rulania, T. 2016. "Impact \& Applications of 3D Printing Technology”, SSRG International Journal of Computer Science and Engineering (SSRG-IJCSE), 3(8).

9. Ukinkar, V.G. and Samvatsar, M. 2012. "Object Detection in Dynamic Background Using Image Segmentation: A Review", International Journal of Engineering Research and Applications (IJERA), 2(3): 232-236.

10. Luan, X., Xie, Y., Ying, L. and Wu, L. 2008. “Research and Development of 3D Modeling”, International Journal of Computer Science and Network Security, 8(1).

11. Kenji, Y., Tomoyuki, M., Takanori, S., Ryutaro, O. and Taiichiro, K. 2010. "3D-Image Technology Electronic Holography System Using 4K Video System”, Journal of the National Institute of Information and Communications Technology, 57(1-2).

12. Zhang, Y. and Zhang, L. 2013. "The Research of 3D Reconstruction Based on Single Image", International Symposium on Computer, Communication, Control and Automation (3CA 2013). 
